\title{
The time to name disoriented natural objects
}

\author{
PIERRE JOLICOEUR \\ University of Waterloo, Waterloo, Ontario, Canada
}

\begin{abstract}
A series of experiments revealed systematic effects of orientation on the time required to identify line drawings of natural objects. Naming time increases as patterns are rotated further from the upright. With practice, however, the effect of orientation is reduced considerably. Furthermore, the reduced orientation effect with practice on a set of objects does not transfer to a new set of objects, suggesting that the acquired ability to reduce the orientation effect is specific to particular patterns. Finally, for departures in orientation from the upright between $0^{\circ}$ and $120^{\circ}$, the magnitude of the orientation effect on identification for patterns seen for the first time is equivalent to that found in a mental rotation task using the same patterns (making left/right decisions about rotated patterns). This final result suggests that novel depictions of a known class of objects may be identified by a process of mental rotation.
\end{abstract}

Psychologists have long been interested in how the orientation of the visual world affects pattern recognition (e.g., Dearborn, 1899; Gibson \& Robinson, 1935; Rock, $1956,1973)$. There are two main reasons for this continued interest in the problem of orientation. First, people have a remarkable ability to compensate for marked changes in the orientation of the visual world in everyday life. For example, tilting one's head $90^{\circ}$ to the side does not cause the world to fragment into a blooming, bustling confusion. The visual world maintains perceptual stability despite large changes in the orientation of the retinal image. It is thus important that any general theory of perception be able to explain how this perceptual stability is achieved. A second reason for the interest in the orientation problem is that different models of object identification make different predictions about how orientation should affect perception. Thus, orientation effects can help us distinguish between various possible models of object identification.

Stored representations of objects in long-term memory can be either orientation-specific or orientationindependent. For example, template models, as discussed in many texts in cognition, postulate orientation-specific representations (e.g., Reed, 1978, 1982; Spoehr \& Lehm-

\footnotetext{
I gratefully acknowledge the support of Stephen Kosslyn and the technical assistance of Avram Fefer and of Scott Bradner and the ComputerBased Laboratory at Harvard for valuable technical support in carrying out Experiment 1. I also thank Derek Besner, Roberta Klatzky, Steven Keele, and anonymous reviewers for comments on earlier drafts of the manuscript, and Donald Clark and Doug Snow for technical assistance. Part of this research was conducted at Harvard University while Pierre Jolicoeur was supported by a graduate scholarship from the Natural Science and Engineering Research Council Canada and by a graduate scholarship from Les Fonds F.C.A.C. Pour L'aide et le Soutien a la Recherche; part was conducted at the University of Saskatchewan, and part at the University of Waterloo. The research was supported by NSF Grant BNS-82-40259 awarded to Stephen M. Kosslyn, and by NSERC Grant A2579 awarded to Pierre Jolicoeur. Requests for reprints should be sent to Pierre Jolicoeur, Department of Psychology, University of Waterloo, Waterloo, Ontario, Canada N2L 3G1.
}

kuhle, 1982). Conversely, many structural models of pattern memory postulate orientation-independent representations. These models often assume that shapes are represented with respect to a frame of reference that depends on the intrinsic properties of the object (such as the axis of maximum elongation), which renders the stored representation independent of the viewing orientation (e.g., Hinton \& Parsons, 1981; Jolicoeur \& Kosslyn, 1983; Marr, 1982; Marr \& Nishihara, 1978). Another frequently suggested possibility is that patterns are represented as combinations of elementary features (e.g., Gibson, 1969; Rumelhart, 1970; see Johnston, 1981, for a recent example of a feature model). In feature models, the stored representations can be either orientation specific or orientation independent, depending on whether the features themselves are orientation specific or independent.

A complete model of pattern recognition, however, must describe not only the nature of memory representations, but must specify also the processes that operate on the inputs to the system and the way in which inputs make contact with stored representations. Predictions concerning the effects of pattern orientation on recognition can be made only if both the representations and processes are taken into account. For example, if patterns are stored using orientation-specific representations, then it may be possible to recognize shapes presented in novel orientations by performing normalization operations on the input to compensate for the orientation difference between the input and the stored representation. Usually, it is assumed that normalization operations take an amount of time that is proportional to the difference between the input and the memory representation along the dimension to be normalized (Dixon \& Just, 1978; Neisser, 1967). Thus, the normalization of orientation should slow recognition time proportionally with the degree of orientation disparity between the input pattern and the stored representation.

Models that postulate orientation-independent representations may also predict effects of orientation on object 
identification. For example, in the model proposed by Marr and Nishihara (1978), the shape of a threedimensional object is represented with respect to a frame of reference that is aligned with the direction of maximum elongation on the object. The resulting representation is independent of the orientation of the object because the frame of reference used to encode the object remains invariant as the object is rotated in space. However, rotating the object may alter the ease with which the viewer can assign the intrinsic or object-centered frame of reference to the object (see Jolicoeur \& Kosslyn, 1983; Marr \& Nishihara, 1978; Warrington \& Taylor, 1973). Thus, while the representation of the object is orientation invariant, the processes that produce that representation may not be completely insensitive to orientation.

The postulated effect of orientation in feature theories also depends on the nature of the processes that map the features onto the stored representations. For example, Hinton (1981) outlined a general scheme in which orientation-specific features are mapped onto orientationindependent representations. In this scheme, the outputs of feature detectors are modulated by mapping units that are tuned to particular orientations. The modulation process acts to compensate for orientation differences between the stored representation and the incoming pattern. By assuming that some mapping units are more likely to be activated than others (e.g., the mapping units for upright and near-upright orientations), the model predicts that orientation could affect the pattern recognition process in a systematic way.

In general, the problems are to discover how patterns are represented in the visual system, and what are the nature and function of the processes that generate and operate on these representations. The response of the visual system to changes in the orientation of patterns presented for identification can be diagnostic in evaluating models of pattern recognition. For example, if pattern recognition proceeds via the extraction of "orientation-invariant features, " then we would expect no effect of orientation on recognition. In contrast, if normalization processes are used, then we should observe systematic effects of orientation on identification.

In light of the above discussion, it is relevant that several recent studies suggest that changes in retinal orientation have little or no effect on the time required to identify simple patterns (Corballis \& Nagourney, 1978; Corballis, Zbrodoff, Shetzer, \& Butler, 1978; Eley, 1982; Simion, Bagnara, Roncato, \& Umilta, 1982, Experiment 3; White, 1980). Based on these studies, it is tempting to conclude that the identification of simple patterns proceeds solely via the extraction of orientation-invariant features.

Eley (1982) investigated two possible reasons for the failure to observe effects of orientation on the time required to identify simple patterns (e.g., Corballis \& Nagourney, 1978; White, 1980). The first possibility is that prior experiments used too few patterns. Because most of the experiments in the literature have used only six characters (three letters and three digits), it is plausible that a set of distinguishing features may have been isolated easily in such a small set of patterns. The second possibility is that alphanumeric characters, by virtue of being highly overlearned patterns, may have become immune to the effects of orientation, but that other simple patterns may not be immune. Eley (1982) included two experimental manipulations designed to address these issues. First, the stimuli consisted of a set of novel simple shapes and therefore were not familiar to his subjects. In the first of the experiment's two phases, subjects learned names (pronounceable nonsense trigrams) for each of the symbols. In the second phase, subjects were required to name the symbols, which could be presented at one of six orientations, as quickly as possible. Two groups of subjects were employed. One group received only enough training in the name-learning phase of the experiment to be able to perform the speeded naming task with relatively few errors. The second group, in contrast, received more extensive training in the name-learning task. Eley's hypothesis was that, if the identification of alphanumeric characters is unaffected by changes in orientation by virtue of their overlearned nature, then perhaps the group with more training in the name-learning phase would be less affected by changes in the orientation of the symbols than the group with only minimal training. There was one other important manipulation in Eley's experiment. Although all the subjects learned to name 20 novel symbols in the first phase of the experiment, half of the subjects performed the speeded naming task with only 5 symbols, and the other subjects were tested on the complete set. Eley hoped that greater effects of orientation would be found in the group tested with 20 shapes than in the group tested with only 5 shapes. With 20 shapes used in the testing phase, Eley reasoned, it should be more difficult for subjects to isolate a set of distinguishing, orientation-invariant features than when fewer shapes could appear. The results in all conditions, however, showed no hint of a systematic effect of orientation on the time required to name the shapes. Based on these results, Eley concluded that the identification of simple shapes usually proceeds via a feature-extraction process, even when the shapes are novel and are drawn from a large set (20 shapes).

There is some evidence, however, that does not support the generality of Eley's (1982) suggestion. Jolicoeur and Landau (1984) presented single alphanumeric characters followed by a pattern mask to subjects, whose task was to report the identity of the character immediately after each presentation. Each of 12 characters was presented in 12 orientations (varying in $30^{\circ}$ steps of rotation in the image plane). The results were unambiguous: Error rate increased linearly with the angular departure of the characters from the normal upright orientation (see also Kolers \& Perkins, 1969a, 1969b).

A number of studies, on the one hand, suggest that orientation has a negligible effect on pattern identification (Corballis \& Nagourney, 1978; Corballis et al., 1978; Eley, 1982; Simion et al., 1982, Experiment 3; White, 1980). 
On the other hand, some studies demonstrate robust effects of orientation on identification (Jolicoeur \& Landau, 1984; Kolers \& Perkins, 1969a, 1969b). These results make it difficult to determine, at an empirical level, what actually are the effects of orientation, if any, which in turn makes it difficult to evaluate the relative merits of various models of human pattern recognition. More empirical work is required, therefore, to discover the way in which orientation affects pattern recognition.

The experiments in this paper investigate how the identification of visual patterns is affected by orientation. All four experiments used line drawings of natural objects as stimuli. Because these stimuli are more complex and less well learned than alphanumeric symbols, I expected that, with these stimuli, orientation effects on identification time would be easier to observe and study than with alphanumeric symbols. The potential importance of complexity is suggested by the observation that pictures of faces are particularly difficult to recognize when they are viewed upside down (Rock, 1973, 1974; see also Koriat \& Norman, 1985). Experiment 1 was designed as a simple demonstration of the effect of orientation on identification time. Experiments 2 and 3 provide further evidence for the basic effect and examine how familiarity with the general class of objects and familiarity with the specific patterns used within an experimental session modulate the orientation effect. The reason for investigating whether familiarity with the patterns alters the orientation effect is that different models of pattern recognition make different predictions concerning the nature and locus of familiarity on the recognition of disoriented patterns.

Consider, for example, how familiarity might operate in a simple template model. Repeated presentation of the same pattern in different orientations could lead to the storage of multiple templates for that pattern, one template for each new orientation of the pattern. These multiple templates could, in turn, reduce the later impact of orientation on recognition. A subject could recognize a disoriented version of the original pattern by matching any one of the pattern's multiple templates, some of which could be closer in orientation than the template for the original orientation.

Familiarity with particular patterns also would be expected to alter the effects of orientation in other models of pattern recognition. For example, in the Marr and Nishihara (1978) model, familiarity could speed up processes that assign the direction of the intrinsic frame of reference to the object. In Hinton's (1981) model, familiarity could change the resting level of mapping units, the resting levels of the canonical object representations, or the strength of connections between mapping units and object representations. These possibilities are discussed further in the General Discussion. Another possibility is that familiarity with patterns could enable subjects to extract a set of orientation-invariant distinguishing features as they become more familiar with a particular set of patterns. With increasing familiarity, subjects could rely more and more on this set of distinguishing features, which would lead to a reduction in the effect of orientation. In general, the models considered here lead to predictions that orientation effects should diminish with repeated testing on disoriented objects. This general prediction was investigated in Experiments 1 to 3 .

Finally, subjects in Experiment 4 were asked to decide whether a disoriented object would face left or right, if it were in the usual upright orientation. Mental rotation of the pattern has been proposed by other researchers as the mechanism used to compensate for disorientation in this sort of task (e.g., Shepard \& Cooper, 1982). The size of the orientation effect in this task is compared with that found in the naming task to determine whether mental rotation could be at the root of the orientation effect on identification time.

\section{EXPERIMENT 1}

Subjects in this experiment were asked to name as quickly as possible drawings of natural objects. There are two main reasons to use drawings of natural objects as stimuli rather than alphanumeric characters. First, effects of orientation may be easier to observe if the shapes to be identified require more time to encode than the time needed to encode alphanumeric characters or the letterlike symbols employed by Eley (1982). For example, if alphanumeric characters can be identified in less than $40 \mathrm{msec}$ of processing time (Jolicoeur \& Landau, 1984; Sperling, 1963), then even a substantial effect of orientation may result in a very small increase in response time. The second reason to use drawings of natural objects is that by using stimuli that subjects can readily identify without prior exposure or learning, the effects of familiarity with the stimuli may be assessed with greater precision. Eley (1982) did not observe a difference in the effects of orientation on identification between subjects who had extended learning on his materials and subjects who had minimal training; however, it is possible that both groups had become familiar enough with the stimuli in the learning phase to overcome the effects of orientation. This possibility is avoided by using stimuli that have not been seen previously by the subjects.

\section{Method}

Subjects. The subjects were 16 Harvard University students who participated in the experiment for pay. All subjects had normal or corrected-to-normal vision, and no subject in this experiment participated in any other experiment in this study.

Materials. The stimuli were 36 watercolor drawings of natural objects photographed onto $35 \mathrm{~mm}$ slides at six orientations (in $60^{\circ}$ steps of clockwise rotation in the plane). The complete list of items is given in Appendix A (see Jolicoeur, Gluck, \& Kosslyn, 1984, for a more detailed description of the objects). Drawings of an additional 8 objects were used in practice trials.

Procedure. The task consisted of naming each object as quickly as possible with the first appropriate name that came to mind. Each subject named all of the objects at every orientation. The stimuli were divided into six blocks of 36 trials. Each object appeared once in each block at one particular orientation. Six objects were presented at each orientation in each block. The stimuli were ordered at ran- 
dom within the above constraints. The order of presentation of blocks was varied from subject to subject using a Latin-square design.

The stimuli were presented using a slide projector and a rearprojection screen. Naming latency was recorded by a microcomputer using a microphone and a voice-activated relay, with timing beginning at the opening of an electronic shutter. The slide remained in view until the subject made a response. A new slide appeared $1 \mathrm{sec}$ after the previous response. Eight trials were used as practice before the first block of trials.

\section{Results}

Naming times for correct responses were averaged over items for each block, each orientation, and each subject. Figure 1 shows the mean naming latency at each orientation for each of the six blocks in the experiment. [Note that the results shown for $0^{\circ}$ and $360^{\circ}$ throughout the paper reflect the same data and are displayed in this way for aesthetic reasons (i.e., to help the reader judge the degree of symmetry about $180^{\circ}$ ); however, the analysis only considered the $0^{\circ}$ data once.] The means were submitted to a repeated-measures ANOVA in which blocks, orientation, and subjects were the factors.

The overall ANOVA revealed two trends in the results that can be seen in Figure 1. First, there was a significant overall effect of orientation on naming time $[F(5,75)$ $=6.03, p<.0001]$. Naming time had a tendency to increase as the stimuli were rotated further from the usual upright, which resulted in a significant linear trend over disorientation $[F(1,15)=23.31, \mathrm{p}<.0003]$. Through-

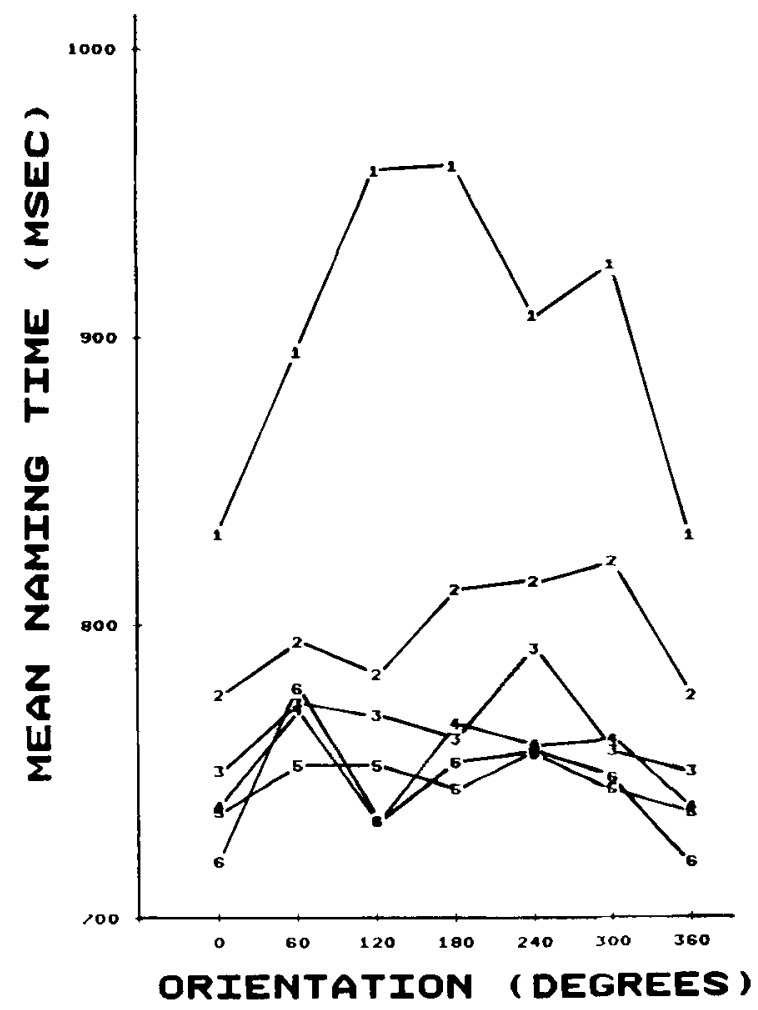

Figure 1. Mean naming time (in msec) in each block at each orientation in Experiment 1. The results for each block are indicated by using the block number as the plotted symbol. out the paper, unless otherwise indicated, the following contrast weights were used to test for the linear increase in response time over disorientation: $-3,-1,1,3,1,-1$, for data at $0^{\circ}, 60^{\circ}, 120^{\circ}, 180^{\circ}, 240^{\circ}, 300^{\circ}$, respectively. This contrast is referred to as the linear trend over orientation, the linear trend over disorientation, or the linear increase for stimuli rotated further from the upright. The second striking aspect of the results is that naming time became shorter with repeated testing, which was reflected in the analysis by a significant effect of blocks $[F(5,75)$ $=34.93, \mathrm{p}<.0001]$.

As can be seen in Figure 1, naming time in the first block of trials increased sharply for objects that were rotated further from the upright. This effect seemed to be reduced with further testing (see especially the results for Blocks 3 to 6). The overall analysis did not reveal this apparent change in the effect of orientation with additional blocks of testing $[F(25,375)=1.15, p>.28]$; however, the change in the linear effect of orientation did vary depending on blocks $[F(5,75)=2.89, p<.02]$. This contrast confirms what can be seen in Figure 1, namely that the tendency for naming time to increase for objects rotated further from the upright has a tendency to decrease with additional testing.

Because of the striking difference between the results for the first block and the results in subsequent blocks, the data for the first block were also analyzed separately. The regular increase in naming times for increasingly disoriented patterns resulted in a significant linear contrast $[F(1,15)=13.19, p<.0025]$, which accounted for $74 \%$ of the variance in naming latency over orientation. The residual variance in naming times in the first block (after the variance for the linear trend was removed) was not significant $[F(4,60)=1.18, p>.32]$. Thus, the overall effect of orientation on naming times in the first block $[F(5,75)=3.65, p<.006]$ was almost entirely due to a linear increase in response time as shapes were increasingly rotated from the upright (see Figure 1).

A second repeated-measures ANOVA on the data for the last five blocks of trials was also computed. In this analysis, subjects, blocks, and orientation were considered as factors. As is evident in Figure 1, subjects named the objects more rapidly with additional practice. This was reflected in the analysis by an overall main effect of blocks $[F(4,60)=6.18, p<.0004]$. The overall effect of orientation in the last five blocks was reliable $[\mathrm{F}(5,75)=3.43$, $\mathrm{p}<.008$ ], and the linear trend in naming times for increasing disorientation was marginally significant $[F(1,15)$ $=4.54, p<.051]$. The effect of orientation in the last five blocks seems to be a result of a consistent advantage of upright patterns over patterns in other orientations.

Errors. The main dependent measure in this study was response time; however, error rates were also measured to ensure that the response time results would not be due only to speed/accuracy trade-offs. Trials in which subjects used an inappropriate name were treated as errors and discarded. Also, trials in which subjects hesitated and emitted vocal sounds (e.g., "hum-m-m") before saying 
the name of an object also were discarded and treated as errors. The percent error rate at each orientation, averaging over blocks, was as follows: $0^{\circ}, 5.0 \% ; 60^{\circ}, 4.2 \%$; $120^{\circ}, 3.6 \% ; 180^{\circ}, 4.7 \% ; 240^{\circ}, 4.3 \% ; 300^{\circ}, 3.0 \%$. These error rates, as well as finer analyses of the error rates, failed to reveal systematic relationships between mean naming time and error rate. Thus, the effect of orientation on naming time was not simply the result of speed/accuracy trade-offs.

\section{Discussion}

Early in testing, the time to name natural objects increases regularly as the orientation of the objects departs further from the standard upright orientation. This result is strongest in the first block of trials when subjects are least familiar with the stimulus materials. The effect of orientation is substantial, amounting to a $129-\mathrm{msec}$ difference between shapes presented at $0^{\circ}$ and $180^{\circ}$ in the first block (see Figure 1).

In contrast with the results in several published reports using alphanumeric or alphanumeric-like characters (Corballis \& Nagourney, 1978; Corballis et al., 1978; Eley, 1982; Simion et al., 1982; White, 1980), this experiment reveals a clear-cut effect of orientation on identification time. Similar results have also been obtained by Shwartz (1981). These results allow us to argue against a general model of pattern recognition based solely on the extraction of "orientation-invariant features."

The effect of orientation decreases dramatically, however, as subjects receive more exposure to the stimuli in the experiment (see Figure 1). This finding suggests that practice or familiarity with the stimuli can attenuate the effect of orientation on pattern recognition. This aspect of the results is explored further in Experiments 2 and 3.

\section{EXPERIMENT 2}

The purpose of this experiment was to replicate and extend one of the main findings of Experiment 1: that the effect of orientation diminishes sharply with repeated testing.

In this experiment, a completely new set of items was used and new subjects were tested in a design similar to that used in Experiment 1. However, there were some other differences between Experiment 1 and this experiment. One difference was that the stimuli in this experiment were black-and-white outline drawings rather than watercolor drawings. In Experiment 1, the color of the various objects could have served as an orientationinvariant cue, and subjects may have used this cue after the first block of trials. If a decrease in the effect of orientation were found again with practice in this experiment, then the results would be evidence that orientationinvariant color cues are not solely responsible for the decreased orientation effect.

Another difference between this experiment and Experiment 1 was that two types of items were included in the experiment: familiar and unfamiliar objects. This manipu- lation was designed to test whether changes in orientation are more pronounced for patterns that are less familiar. This possibility was suggested by the pattern of results found in Experiment 1 and was also hypothesized by Eley (1982). In the first block of trials in Experiment 1, there was a robust effect of orientation on identification time, whereas in later blocks the effect was reduced. Perhaps subjects became familiar with the stimuli with repeated testing, which may have reduced the effect of orientation. There are, however, two aspects to the notion of familiarity: the familiarity of subjects with the general class of objects they are trying to identify, and the familiarity with the specific depictions used to represent a particular category of objects (i.e., the actual stimuli used). By including depictions of objects that differ in preexperimental familiarity, this experiment tested whether orientation effects are more pronounced for unfamiliar objects in a situation in which subjects have equal practice with the actual pictures used in the experiment.

\section{Method}

Subjects. The subjects were 12 University of Saskatchewan undergraduates with normal or corrected-to-normal vision who par ticipated in the experiment without pay. No subject in this experiment participated in any other experiment in this paper.

Materials. The stimuli were 72 black-and-white outline drawings taken from Snodgrass and Vanderwart (1980). The items in the drawings belonged to two subsets that differed in degree of familiarity, according to subjects' ratings. The ratings, reported by Snodgrass and Vanderwart (1980), were on a 5-point scale in which a 1 indicated very unfamiliar and a 5 indicated very familiar. Subjects were asked to rate the familiarity of the pictures "according to how usual or unusual the object is in your realm of experience," and "the degree to which you come into contact with or think about the concept." (Snodgrass \& Vanderwart, 1980, p. 183). The 36 familiar items had familiarity ratings ranging from 3.35 to 4.82 with a mean of 4.33 , whereas the unfamiliar items had ratings rang ing from 1.45 to 2.48 with a mean of 2.01. The name and familiarity rating of each item are listed in Appendix B. The actual drawings can be seen in Snodgrass and Vanderwart (1980).

The drawings were photocopied onto a translucent Mylar film using a high-quality photocopier and then mounted onto $35-\mathrm{mm}$ slides. Each drawing was mounted at six different orientations in $60^{\circ}$ steps of clockwise rotation starting at $0^{\circ}$. A set of 12 additional items ( 2 items at each orientation) was also prepared for practice trials.

Procedure. The stimuli were projected onto a rear-projection screen, and the task was to name the displayed object as quickly as possible with the first appropriate name that came to mind. The slide remained in view until the subject made a response or until 4,000 msec elapsed without a response. A new slide was presented 1,000 msec after the removal of the previous slide. Naming time was measured as in Experiment 1.

The stimuli were divided into six blocks of 72 trials. Each object was included once in each block; thus there were 36 familiar and 36 unfamiliar objects in each block. Furthermore, 6 familiar and 6 unfamiliar objects were presented at each of the six orientations in each block. Each block was further subdivided into three subblocks of 24 trials that included an equal number of familiar and unfamiliar objects at each of the six orientations. The stimuli were ordered at random within the above blocking constraints.

Each subject saw three blocks of trials preceeded by 12 practice trials. The order of blocks was counterbalanced across subjects using a Latin-square design such that each block was seen equally often in each of the three positions in the testing order. 


\section{Results}

Naming time. The naming times for correct responses were averaged over items for each orientation, block, level of familiarity, and subject. These means were submitted to a repeated-measures ANOVA in which subjects, familiarity, orientation, and blocks were considered as factors.

The mean naming time at each orientation in each block is displayed in Figure 2. A number of results are immediately apparent. First, there is a strong effect of orientation on mean naming time $[F(5,55)=18.42, p<.0001]$, in which naming time tends to increase linearly as objects are rotated further from the upright $[F(1,11)=$ $50.32, \mathrm{p}<.0001]$. Second, naming time decreases with each block of testing $[F(2,22)=31.37, p<.0001]$. And third, the effect of orientation depends on blocks $[F(10,110)=2.40, p<.013]$. As can be seen in Figure 2, the steepness of the linear increase in naming time decreases across blocks of testing $[\mathrm{F}(1,11)=26.13$, $\mathrm{p}<.0004]$ (as tested by the interaction of blocks with the linear trend across disorientation). These results replicate the main results seen in Experiment 1: a strong effect of orientation early in the experiment that tended to diminish with repeated testing on the same items.

The mean naming time at each orientation for familiar and unfamiliar objects is depicted in Figure 3. While un-

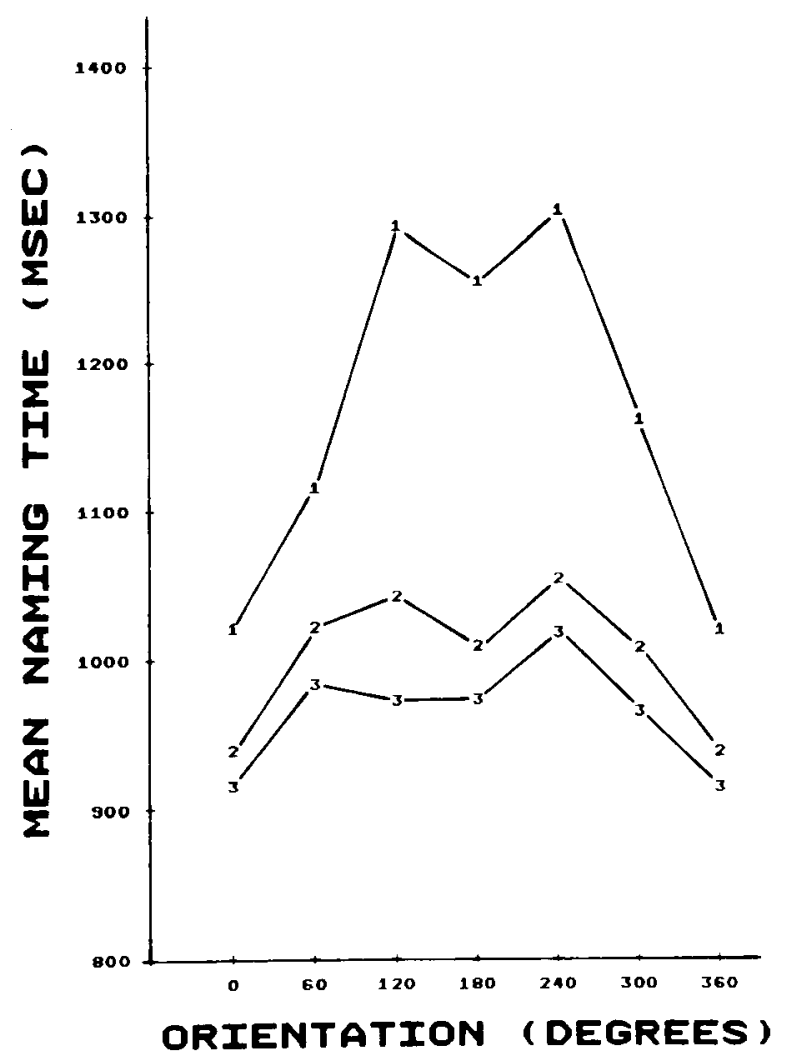

Figure 2. Mean naming time (in msec) in each block at each orientation in Experiment 2. The results for each block are indicated by using the block number as the plotted symbol.

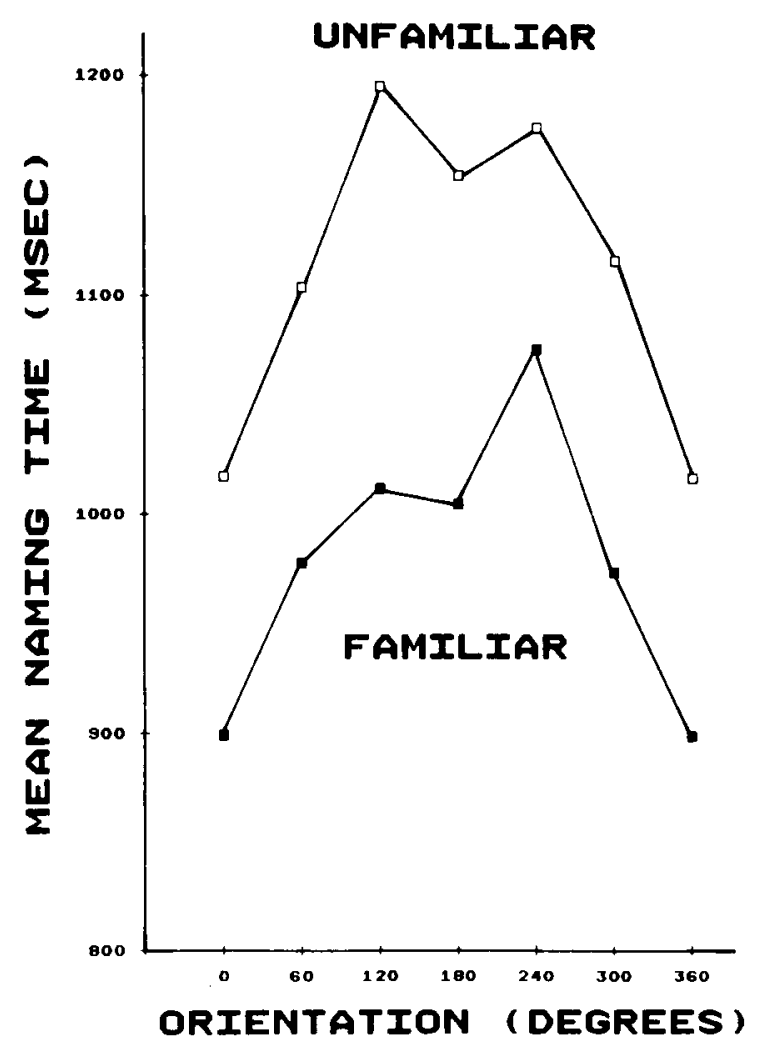

Figure 3. Mean naming time (in msec) at each orientation for familiar objects (filled squares) and unfamiliar objects (open squares) in Experiment 2.

familiar objects were named more slowly than familiar objects at all orientations $[F(1,11)=54.95, p<.0001]$, the effect of orientation was very similar for these two sets of items. The analysis does not reveal any differences in the effect of orientation between familiar and unfamiliar objects $(F<1)$ both for the overall interaction and for the difference between linear trends. Thus, familiarity with the general class of objects, as indexed by Snodgrass and Vanderwart's (1980) ratings, is not as important in reducing the effect of orientation as familiarity with the specific set of items used in the experiment.

There is only one other significant effect in the analysis: Although mean naming time decreased from block to block for both familiar and unfamiliar objects, this pattern was more marked for unfamiliar than for familiar objects (familiar objects: 1098, 959, $914 \mathrm{msec}$ for blocks 1,2 , and 3 , respectively; unfamiliar objects: 1288 , $1067,1030 \mathrm{msec}$ for blocks 1,2 , and 3, respectively) $[F(2,22)=4.47, p<.024]$. Similar results have been obtained by Jacoby and Dallas (1981) in a perceptual recognition experiment in which the interaction was between word frequency and repetition.

Errors. Names that were clearly erroneous for a given object were treated as errors (e.g., calling a zebra a clock). In addition, hesitations, prenaming vocalizations (e.g., "hum-m-m"), and other spurious triggerings of the voiceactivated relay were all treated as errors. The error rates 
for blocks, orientation, and familiarity were examined to determine whether the naming latency results could be due to speed/accuracy trade-offs. The percent error rate at each orientation, averaged over blocks, are as follows: $0^{\circ}, 3.2 \% ; 60^{\circ}, 2.8 \% ; 120^{\circ}, 2.5 \% ; 180^{\circ}, 3.5 \% ; 240^{\circ}$, $3.9 \% ; 300^{\circ}, 3.0 \%$. These error rates, as well as finer analyses of the error rates failed to reveal systematic relationships with naming time. Thus, the effect of orientation on naming time is not simply the result of speed/accuracy trade-offs.

\section{Discussion}

The results are clear-cut: First, objects rotated further from the usual upright orientation are named more slowly than less rotated objects; second, the effect of orientation is strongest in the first block of trials and decreases with practice. These results confirm the results of Experiment 1 and extend them to monochromatic line drawings of natural objects. Given that similar results have been obtained in two separate experiments, with different materials and subjects, there is reasonably good evidence that the effects of disorientation on the identification of disoriented patterns are reduced with practice.

Although the effect of orientation decreases over testing blocks, naming time for objects rated as unfamiliar is not affected more by disorientation than naming time for objects rated as familiar. These findings suggest that the decrease in the effect of orientation over blocks is due to practice with the particular slides used in the experiment rather than to a general tendency for depictions of familiar concepts to be immune from orientation effects. However, it could be argued that the particular measure of familiarity used in this experiment is not the best possible one to test the notion that familiarity with a class of patterns reduces the effects of orientation. For example, it may be that ratings of familiarity with the appearance of the objects in a class would have been better than the frequency or usualness of the class. More research would be required to test this hypothesis. While this notion is not investigated here, the notion that practice with particular pictures is responsible for the reduction in the orientation effect is studied more directly in the next experiment.

Although the general pattern of results found in this experiment is similar to that found in Experiment 1, there are a number of differences worthy of mention. For example, naming time in this experiment is slower overall than in Experiment 1. There are several possible explanations for this difference, including the notions that objects in Experiment 1 were more familiar, or that the names of the objects used in Experiment 1 were more easily retrieved than in the present experiment. Another possibility is that the presence of color information in Experiment 1 may have facilitated the identification of the objects and reduced naming time.

There are three additional differences between the results of Experiments 1 and 2. First, the effect of orientation in the first block of trials is more pronounced in this experiment than in Experiment 1 (a 102-msec difference between $0^{\circ}$ and $120^{\circ}$ in Experiment 1 compared with a 280-msec difference in Experiment 2). Second, the orientation effect seems to decrease more sharply with practice in Experiment 1 than in the present experiment. Both of these differences may be accounted for by noting that different stimuli were used in the two experiments: colored drawings in Experiment 1 and black-and-white drawings in Experiment 2. The color information is orientation-invariant, which may have helped subjects to identify the objects despite the rotation of the images. Furthermore, once all the objects had been seen once, the presence of distinctive color information may have contributed to the reduction in the orientation effect in Experiment 1. The third difference is that, in Experiment 2, the mean naming time for objects presented at $180^{\circ}$ was much smaller than would have been expected by linearly extrapolating from the results across the other orientations (see Figure 2). Other researchers have observed similar results (e.g., Corballis et al., 1978; Dearborn, 1899; Koriat \& Norman, 1984, 1985; Rock, 1973; Rock, Di Vita, \& Barbeito, 1981). This difference may be due to the presence of some objects in the stimulus set that are nearly up-down symmetric (e.g., the drawing of a television set), and/or may reflect the fact that left/right relations are preserved in objects rotated $180^{\circ}$ (Rock, 1973). In any case, this aspect of the results could only lead us to underestimate the effect of orientation on naming time, and thus we can be confident that orientation does have a strong effect on identification time.

\section{EXPERIMENT 3}

In this experiment, we examined more directly the notion that testing on a specific set of items is responsible for the reduction in the effect of orientation observed in $\mathrm{Ex}$ periments 1 and 2 . Two sets of items were used. In the first five blocks of testing, subjects named objects in one of the two sets of items; in the sixth and final block, they named objects in the other set of items. If, in the first five blocks, subjects acquired a general ability to overcome effects of orientation on identification, then this ability should transfer to the new set of items used in the last block of the experiment. If, on the other hand, subjects learned something that was specific to the particular set of slides/objects used in the first five blocks, then this learning should not transfer to the items used in the final block.

\section{Method}

Subjects. The subjects were 12 University of Saskatchewan and 24 University of Waterloo undergraduates with normal or correctedto-normal vision who participated in the experiment without pay No subject in this experiment participated in any other experiment in this paper.

Materials. The materials were identical to those used in Ex periment 2.

Procedure. The task and experimental setup were identical to those used in Experiment 2; however, the stimuli were arranged 
differently. The stimuli were divided into two sets of items, with an equal number of familiar and unfamiliar items in each set. There were 36 objects in each set, Set A and Set B. The items in each set were divided further into six blocks of items composed exclusively of items from a given set, as described in Experiments 1 and 2. This procedure ensured that each item in a set occurred once in each of the six blocks for that set of items, and that six objects were presented at each orientation in each block.

Half of the subjects were tested on five consecutive blocks of items drawn exclusively from Set $A$, and they were then tested on one additional block of items drawn exclusively from Set B. The other half of the subjects were tested first with items from Set B and later with items from Set A. The order of blocks within each half of testing was counterbalanced over subjects using a Latinsquare design to ensure that each block occurred equally often in each possible position of the blocks in the testing order over every 12 subjects.

\section{Results}

Naming time. Two analyses are presented in this section. In the first analysis, mean response times were averaged across items in each block at each orientation for each subject (the Subject analysis). In the second analysis, naming times were averaged across subjects for each item at each orientation in each block (the Item analysis).

\section{Subject Analysis}

The naming times for correct responses in each block at each orientation and for each subject were averaged. These means were submitted to a repeated-measures ANOVA in which subjects, blocks, and orientation were considered as factors.

The mean naming time in each block for each orientation is displayed in Figure 4. Mean naming time again depends on orientation $[F(5,175)=18.57, \mathrm{p}<.0001]$, with a strong linear trend over departure from the upright $[F(1,35)=55.82, p<.0001]$. As in the first two experiments, mean naming time also depends on blocks $[\mathrm{F}(5,175)=123.82, \mathrm{p}<.0001]$.

However, the overall block $\times$ orientation interaction is not significant $[F(25,875)=1.20, p>.22]$. Two contrasts, however, suggest that the effect of orientation does diminish across blocks, and that this effect is specific to the items seen in the first set of slides. The first contrast compares the slopes of the orientation effects in Block 1 and Block 6, and indicates that the orientation effect was equally strong in both blocks $(F<1)$. This comparison suggests that there is no transfer of practice from the first set of items to the second set. The second test compares the average slope of the orientation effect in Blocks 1 and 6 with that in the average of Blocks 4 and $5[F(1,35)=$ $3.95, \mathrm{p}<.055$ ]. This comparison is between two blocks in which slides are seen for the first time (Blocks 1 and 6) with performance in blocks in which particular objects have been seen a number of times previously (i.e., at the end of training with the first set of slides-Blocks 4 and 5). As can be seen in Figure 4, there is little to suggest that whatever reduction in the slope of the orientation effect occurred from Blocks 1 to 5 transfers to the new items in Block 6. In fact, if anything, the orientation effect seems larger in the last block than in the first.

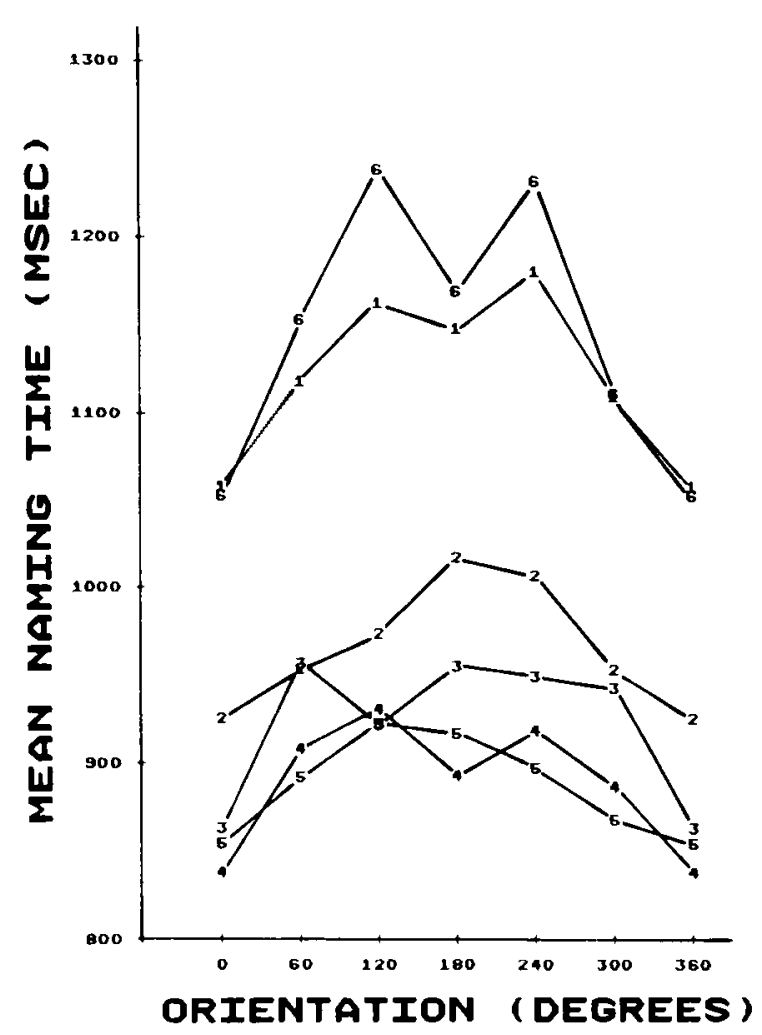

Figure 4. Mean naming time (in msec) in each block at each orientation in Experiment 3. The results for each block are indicated by using the block number as the plotted symbol.

\section{Item Analysis}

The naming times for correct responses in each block at each orientation and for each item were averaged across subjects. These means were submitted to a repeatedmeasures ANOVA in which items, blocks, and orientation were considered as factors. Because only three subjects responded to any one item in a given block at a particular orientation, two of the items had at least one cell with no correct response. These two items were excluded from the analysis, and thus the present analysis is based on 70 items.

As in the Subject analysis, there is a large overall effect of orientation $[F(5,345)=16.24, p<.0001]$, with a strong linear component $[\mathrm{F}(1,69)=54.43, \mathrm{p}<.0001]$. The reduction in naming time across blocks is also reflected in this analysis $[\mathrm{F}(5,345)=87.12, \mathrm{p}<.0001]$.

As in the first two experiments, the effect of orientation is mitigated by blocks $[\mathrm{F}(25,1725)=1.65, \mathrm{p}<$ $.023]$, as is the linear trend in orientation $[F(5,345)=$ $3.12, \mathrm{p}<.01]$. The interaction between blocks and the linear trend in orientation was further examined in a series of contrasts. As in the Subject analysis, there is no indication that the effect of orientation differed between Blocks 1 and $6(F<1)$. Unlike in the Subject analysis, however, the difference between the linear trend in orientation is clearly reliable between Blocks 5 and $6[\mathrm{~F}(1,69)$ $=5.47, \mathrm{p}<.023]$, and this trend is marginally significant between Blocks 1 and $5[F(1,69)=3.63, p<.062]$. 
Together, these results confirm that the effect of orientation is reduced after repeated testing with a fixed set of items, as was found in Experiments 1 and 2. Furthermore, there is no transfer of this reduction in the orientation effect to a new set of items (in Block 6), as can be seen in Figure 4.

Finally, the Item analysis allows us to test again the notion that rated familiarity (from Snodgrass \& Vanderwart, 1980 ) with the objects might mitigate the orientation effect. In this test, the results for Blocks 1 and 6 were averaged because the objects were seen for the first time in these blocks and because the orientation effect was not significantly different across the two blocks. A linear contrast was computed for each item and correlated with the rated familiarity for that item. The correlation was $r=$ $-.185(\mathrm{p}>.12)$, again indicating that the rated familiarity of the item is not a very good predictor of the magnitude of the orientation effect upon initial viewing.

Errors. Errors, defined as in Experiment 2, were examined for possible speed/accuracy trade-off counterexplanations of the mean naming-time results. The percent error rates across orientation are as follows: $0^{\circ}, 2.9 \%$; $60^{\circ}, 4.4 \% ; 120^{\circ}, 5.3 \% ; 180^{\circ}, 5.1 \% ; 240^{\circ}, 4.6 \% ; 300^{\circ}$, $5.0 \%$. These results and those of finer analyses provide no evidence that the mean naming-time results are due to speed/accuracy trade-offs.

\section{Discussion}

The results support two hypotheses concerning the effect of orientation on the time to identify objects presented visually. First, the effect of orientation on naming time is attenuated with practice on a fixed set of objects, and second, this practice effect does not transfer to new objects. It appears that the learning which allows subjects to overcome the orientation effect with practice is quite specific in nature and is not a general ability to identify disoriented objects.

Finally, systematic orientation effects were obtained in Experiments 2 and 3 even after several blocks of testing with the same objects. This aspect of the results provides strong support for the notion that pattern recognition mechanisms used to identify natural objects are sensitive to changes in orientation.

\section{EXPERIMENT 4}

The results of the previous three experiments demonstrated that the time to identify disoriented natural objects increases with increasing disorientation. Research on mental rotation has also revealed a similar relationship. The issue addressed in Experiment 4 was whether the orientation effects observed in the identification of objects could be attributable to mental rotation.

Several researchers have argued that mental rotation is used to make left/right decisions about rotated visual stimuli (e.g., Corballis, Zbrodoff, \& Roldan, 1976; Shepard \& Cooper, 1982; Shepard \& Metzler, 1971). The effect of orientation in this type of experiment when al- phanumeric characters are used typically is much more pronounced than the effect of orientation on identification time (White, 1980). One may wonder whether the orientation effects observed on the time to identify objects is large enough to be due to mental rotation. This question was addressed in this experiment by requiring subjects to make left/right decisions with rotated objects and by estimating the rate of mental rotation. These results are then compared with those obtained in Experiment 3 with the same objects.

\section{Method}

Subjects. The subjects were 12 University of Saskatchewan undergraduates who participated in the experiment without pay. All subjects had normal or corrected-to-normal vision, and no subject had been in any of the previous experiments reported in this paper.

Materials. The stimuli were 36 objects drawn from the pool of 72 used in Experiments 2 and 3 (see Appendix B). Only objects that faced clearly to the left or right were included (e.g., a side view of an animal). As in the previous experiments, each object was tested at each of six orientations.

Procedure. The stimuli were presented as in the previous experiments, and the task was to decide, as quickly as possible, whether each object faced left or faced right when rotated to the usual upright orientation. A subject indicated the response by pressing one of two response keys with the index finger of either hand. The subject was instructed to press the right key if the object faced to the right and the left key if the object faced to the left. Response time was measured from the onset of the picture to the subject's key press.

The stimuli were divided randomly into six blocks of 36 trials with the constraint that half of the objects in each orientation in each block face left and half face right. Every object was used once in each block.

For half of the subjects, each object faced left in three trials and faced right in three trials (at different orientations). For the other half of the subjects, the slides that faced left for the first half of the subjects now faced right, and the slides that faced right were changed to face left. Overall, each object was tested in each orientation and each left/right response an equal number of times.

All subjects were tested on six blocks of trials and the order of blocks was counterbalanced over subjects using a Latin-square design. The experiment began with 12 practice trials.

\section{Results}

Response times. For each subject, we averaged the response times for correct responses in each orientation, in each block, and for objects facing in each direction. These means were submitted to a repeated-measures ANOVA in which subjects, direction (left/right), blocks, and orientation were considered as factors.

The mean response time at each orientation in each block is displayed in Figure 5. Two results are immediately apparent. First, there is a large and consistent effect of orientation on decision times $[F(5,55)=37.83$, $\mathrm{p}<.0001]$. Decision time has a strong tendency to increase linearly as the objects are rotated further from the upright $[F(1,11)=66.13, p<.0001]$. Second, response time decreases across blocks $[\mathrm{F}(5,55)=10.98, \mathrm{p}<.0001]$. The mean response time in each block is $952,826,830$, 812,757 , and $742 \mathrm{msec}$, for Blocks 1 to 6 respectively.

Unlike the results of the previous experiments, however, there is no tendency for the effect of orienta- 


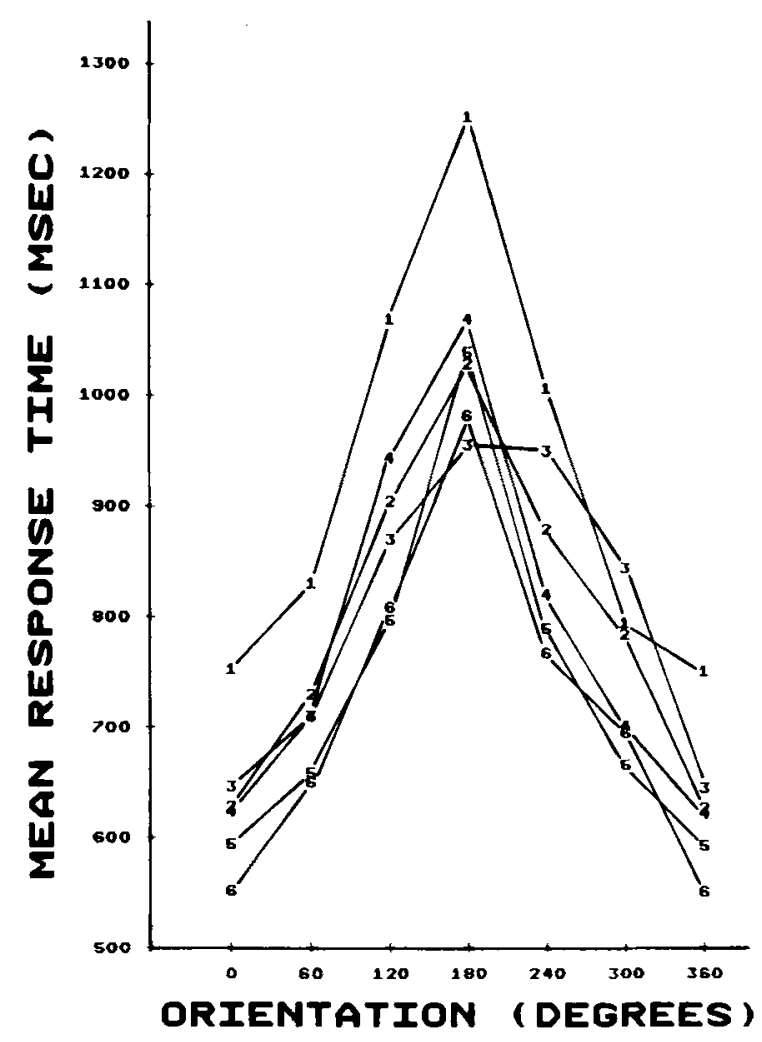

Figure 5. Mean response time (in msec) in each block at each orientation in Experiment 4. The results for each block are indicated by using the block number as the plotted symbol.

tion to decrease over blocks ( $F<1)$. These results suggest that left/right decisions require mental rotation throughout the experiment. Because each object faced left and right an equal number of times for each subject, identifying the object would not allow subjects to make the left/right decision. However, identifying the objects more quickly could help subjects decide in what direction to mentally rotate the stimulus, which could account for the decrease in overall response time across blocks in the experiment.

While "left" and "right" responses take the same amount of time (left, $817 \mathrm{msec}$; right, $823 \mathrm{msec}$ ), there is an overall interaction between left and right responses and orientation $[F(5,55)=2.87, p<.023]$. Left responses are slightly faster than right responses for objects rotated $60^{\circ}$ or $120^{\circ}$ clockwise, and right responses are slightly faster than left responses for objects rotated $240^{\circ}$ clockwise. The linear trend over orientation, however, is not different for left and right responses $(F<1)$, indicating that the effect of orientation is equally marked for left and right trials overall. For this reason, the interaction between response (left/right) and orientation is not discussed further.

There are no other significant effects in the analysis (p > .31 in all cases).
Errors. The percent error rates, averaged over blocks, are as follows: $0^{\circ}, 2.8 \% ; 60^{\circ}, 2.5 \% ; 120^{\circ}, 5.3 \% ; 180^{\circ}$, $18.8 \% ; 240^{\circ}, 5.8 \% ; 300^{\circ}, 2.1 \%$. An inspection of these error rates and finer analyses of the error rates reveal no evidence for a speed/accuracy trade-off account of the response time data.

There is one striking characteristic of the error rates, as can be seen in the list above: The error rate for objects presented at $180^{\circ}$ is much larger than the error rates for the other trials (including objects presented at $120^{\circ}$ or $240^{\circ}$ ). One explanation for these results hinges on the notion of stimulus-response compatibility (Fitts \& Seeger, 1953). If subjects could identify the direction in which the objects pointed with respect to the displayed orientation before mental rotation, then this identification could have led to a tendency to respond with the wrong direction. At $180^{\circ}$ the objects point in the direction exactly opposite the direction pointed at $0^{\circ}$. Perhaps the incompatibility between the correct response and the pointing direction of the stimulus at $180^{\circ}$ caused the sharp increase in error rates observed at this orientation. Another possibility is that some subjects rotated the objects out of the image plane by "flipping" the objects from top to bottom, instead of rotating the objects in the image plane; this tendency may be greatest for upside-down objects. This strategy would produce the wrong response, which may account for the high error rate at $180^{\circ}$.

Effect of orientation in naming versus left/right decisions. In this section the orientation effects in the left/right task are compared with those in the naming task in Experiment 3. The question is whether the magnitude of the orientation effect in the naming task initially is equivalent to the orientation effect in the left/right task.

The data used for the item analysis in Experiment 3 were also used here. Of the 70 items that were used in the item analysis in Experiment 3, 34 were also in Experiment 4 ( 2 items were rejected, as in Experiment 3, because there was at least one cell in the design with no correct response in the naming task). The analyses presented here are based on results for these 34 items.

The results from the first and last blocks of trials in Experiment 3 are included in this analysis (blocks in which the stimuli were seen for the first time). The results from all six blocks in Experiment 4 are used to estimate the magnitude of the orientation effect for each item because there is no significant effect of blocks in this experiment.

In this analysis, Items was a random factor with repeated measures on Task (naming vs. left/right) and on Orientation. Figure 6 displays the mean response time at each orientation for each task. Naming the objects takes longer than deciding whether they face left or right $[F(1,33)=60.72, p<.0001]$. Orientation strongly affects response times $[F(5,165)=37.79, p<.0001]$, and the effect of orientation depends on the task $[F(5,165)=$ $5.34, \mathrm{p}<.0002]$. The linear trend over disorientation is also very strong $[F(1,33)=137.90, p<.0001]$. As 


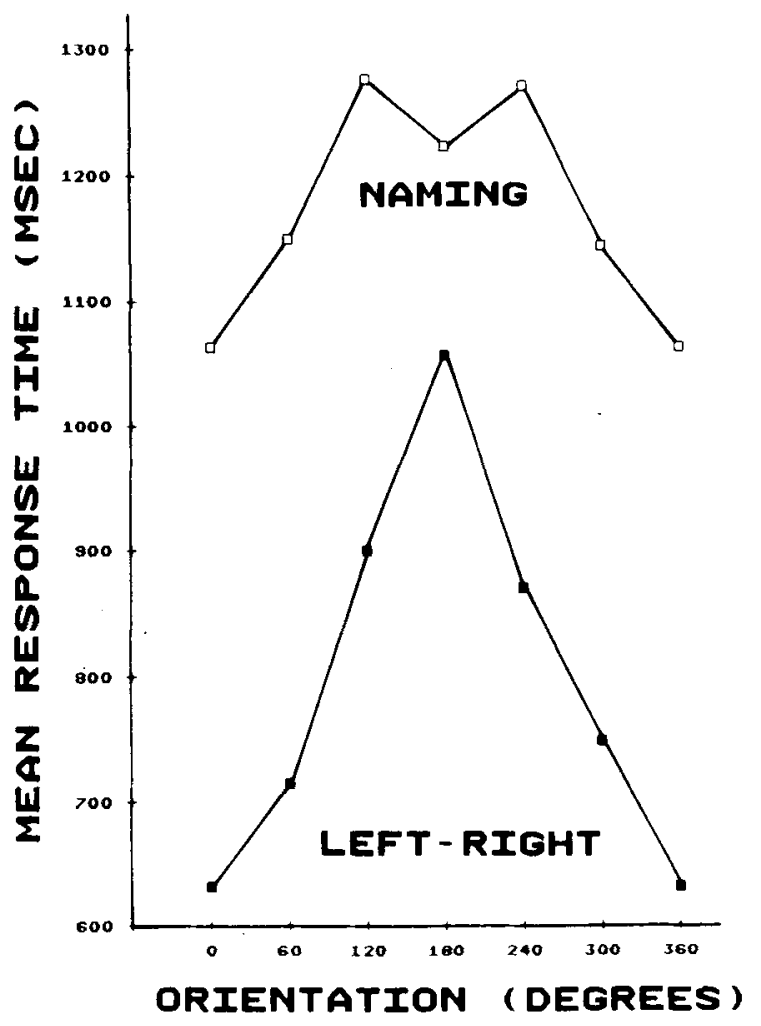

Figure 6. Mean response time (in $\mathrm{msec}$ ) at each orientation in the left-right task (Experiment 4 , filled squares) and in the naming task (Experiment 3, open squares).

is evident in Figure 6, when the results from all orientations are included in the computation of the linear trend, the slope of the orientation effect is steeper in the left/right task than in the naming task $[\mathrm{F}(1,33)=10.68, \mathrm{p}<$ .0026].

Finally, it is also instructive to compare the slope of the orientation effect across the two tasks, but not including the results for the $180^{\circ}$ orientation. There are two reasons to exclude the results for $180^{\circ}$ : First, the dip in the orientation function for the naming task suggests that these data may reflect processes that do not influence the results for other orientations to the same degree. Second, the $180^{\circ}$ results from the left/right task are also somewhat unusual (recall that the error rate at $180^{\circ}$ in the left/right task is five times larger than the average error rate at the other orientations). Thus, in this case also, the results for $180^{\circ}$ trials seem somewhat unlike results for other orientations. Although the linear effect of orientation is highly significant even when the results for $180^{\circ}$ are not included $[F(1,33)=82.70, p<.0001]$, the slope of the orientation function over disorientation does not differ across the two tasks $(F<1)$ (the following contrast was used in the above two tests: $-6,-1,4,0,4,-1$, for $0^{\circ}, 60^{\circ}, 120^{\circ}$, $180^{\circ}, 240^{\circ}$, and $300^{\circ}$, respectively). Thus, it appears that the larger effect of orientation on left/right decisions than on naming in the overall analysis is entirely due to the inclusion of the results from $180^{\circ}$ trials.

\section{Discussion}

The results of this experiment are as expected if subjects perform mental rotation to make left/right decisions about rotated objects. Mean response time increases sharply as the objects are rotated further from the usual upright orientation. Furthermore, the magnitude of the orientation effect in this experiment (425-msec difference between $180^{\circ}$ and $0^{\circ}$ ) is comparable to that obtained in earlier mental rotation studies that used two-dimensional stimuli (Shepard \& Cooper, 1982).

The main purpose of this experiment was to provide a set of results with which to compare the size of the orientation effect on object identification. Given that the items used in this experiment form a subset of the items used in Experiment 3, a direct comparison of response times for these items across the two tasks is possible. If all orientations are considered, then the slope of the orientation effect is larger in the left/right task than in the naming task. However, this analysis includes results from the $180^{\circ}$ trials, which seem to be significantly different than the other orientations in both tasks. In the naming task, there is a dip at $180^{\circ}$, indicating that this orientation is not as difficult as one would expect by extrapolating from the other orientations. In the left/right task, however, the $180^{\circ}$ trials seem to be more difficult than for the other orientations, as indicated by the anomalously high error rate at this orientation. For these reasons, I also compared the size of the orientation effect across the two tasks, excluding the results from $180^{\circ}$ trials. In this comparison, there was no hint of a difference in the magnitude of the orientation effect on response times between naming and left/right decisions.

If the left/right task required the use of mental rotation, then the results suggest that naming natural objects seen for the first time also involves mental rotation (for orientations between $0^{\circ}$ and $120^{\circ}$ ).

\section{GENERAL DISCUSSION}

The results of the series of experiments reported in this paper allow us to make a number of observations about the way people identify disoriented natural objects. First, the time required to identify disoriented visual patterns is dependent on the orientation of the pattern. In every experiment, identification time increased with greater departure from the upright (from $0^{\circ}$ to $120^{\circ}$ ). Second, early in testing with a particular set of rotated objects, the effect of orientation on identification time is equivalent with that found in a mental rotation task (for orientations between $0^{\circ}$ and $120^{\circ}$, at least). Thus, mental rotation may be involved in the identification of disoriented natural objects seen for the first time. Third, the effect of orientation on identification decreases with practice on a given set of materials, but this decrease does not transfer to new objects. 
The fact that the orientation effect on recognition decreases with practice allows us to account for the large number of studies reporting relatively small orientation effects on the identification of alphanumeric characters (e.g., Corballis et al., 1976; White, 1980; for a review see Jolicoeur \& Landau, 1984). Furthermore, if we assume that the reduction in the orientation effect requires less practice when the stimuli are relatively simple, then we may account for Eley's (1982) finding that orientation did not affect significantly the time to name newly learned shapes. Despite Eley's procedure, which attempted to create two groups of subjects differing on training with his novel letter-like symbols-one group with minimal training and one with moderate training-it is possible that both groups in fact received enough exposure to the stimuli to greatly reduce orientation effects that may have been present initially.

While the effect of orientation is reduced after practice, rotated objects nonetheless required more time to process than upright objects in every experiment reported here. Furthermore, even in the case of alphanumeric characters, which are highly overlearned stimuli, Jolicoeur \& Landau (1984) demonstrated a linear effect of orientation on identification errors, and this effect showed no signs of diminishing with further practice. Thus, while training reduces the initial effects of orientation dramatically, as is probably the case for alphanumeric characters, such training does not completely eliminate the influence of orientation (Jolicoeur \& Landau, 1984; Kolers \& Perkins, 1969a, 1969b; see also Koriat \& Norman, 1984, 1985).

How do the different models account for the initial recognition of a pattern seen in a novel orientation? In a template model, we can suppose the use of a normalization operation (Dixon \& Just, 1978; Neisser, 1967). In this view, we would expect a linear effect of orientation on naming time, especially on initial viewing. Normalization could be achieved initially by mental rotation which is not contradicted by the results (for orientations between $0^{\circ}$ and $120^{\circ}$, at least).

In the Marr and Nishihara (1978) model, effects of orientation are more problematic. In their model, the first step in the identification process is the assignment of the direction of the main axis of a frame of reference, which is determined by some salient property of the object. For example, suppose that the main axis is aligned with the direction of maximum elongation of the object. If all objects were elongated along the same direction (a "standard" direction of elongation), there would be no problem. For example, we could assume that the model always attempts to assign the direction of the main axis along the standard direction and that increasing the size of the deviation from the standard direction leads to increasingly long delays in the selection of the direction of the axis. However, objects are longest along different directions and thus there is no "standard" orientation for the main axis of the frame of reference. Given that the drawings of the objects used in the experiments described here did not employ unusual views of the objects (see Warrington \& Taylor, 1973), the assignment of the orientation of the main axis should have been equally easy at all orientations. Thus, it is not clear why linear (or even monotonic) effects of orientation should be expected.

In Hinton's (1981) model, the output of feature detectors (in retinal coordinates) is modulated by mapping units that compensate for the disorientation of the pattern presented on the retina. The features are mapped into another set of features whose orientation is fixed with respect to canonical descriptions of objects built up from the mapped features. In this model, the initial orientation effects can be accounted for by supposing that the mapping units are differentially easy to activate, with the most easily activated units corresponding with the upright and the least easily activated units with $180^{\circ}$. One way of achieving a graded bias from upright to upside-down orientations is to suppose that mapping units have resting levels of activation that are inversely proportional to their corresponding orientation, which could result in part from differential experience with different orientations. In this view, stimuli in unusual orientations would be identified more slowly than upright stimuli because the mapping units for these orientations require more activation before they can reach a level at which they produce a stable interpretation of the input.

Feature models postulating only orientation-invariant features have difficulty in accounting for the result if no form of normalization is assumed. Clearly, if recognition is based entirely on the extraction of orientation-invariant features, then patterns should be recognized equally quickly in any orientation. The results argue strongly against this possibility.

Not only must we account for the initial effect of orientation on identification time, we also must account for the reduction in this effect with practice and for the specificity of this reduction. In models postulating templates, a different view of a particular object could be stored after each block of testing. Although no single object was repeated exactly in the present experiments (i.e., the same object was never shown at the same orientation twice), after several blocks it would become increasingly likely that one of the earlier orientations of the pattern would be close to one in a later block. It would then be possible to identify this pattern by normalizing it to one of the previously identified views rather than by normalizing to the upright.

In Marr and Nishihara's (1978) model, repeated presentations could facilitate the speed of assignment of the object-centered frame of reference used to encode particular objects, which could result in a reduced-orientation effect. As discussed earlier, however, this model has difficulty in accounting for the systematic orientation effect on original viewing.

In Hinton's (1981) model, prior exposure of a stimulus could lead to the strengthening of connections between mapping units and particular canonical representations. However, this account has difficulty with the fact 
that exact repetitions of an object at a given orientation never occurred in the experiments described in this paper. Another way to account for the reduced orientation effect is to suppose that the resting levels of the mapping units become more similar with repeated exposures to rotated stimuli, which would result in more equal response times across different orientations. However, this approach cannot explain why the reduced orientation effect was specific to particular objects. If the reduced orientation effect was due to a change at the level of mapping units, then orientation effects would have been reduced for all stimuli, including new objects. We can do better to assume instead that the resting levels of the canonical object representations change with practice. This approach has the virtue that it explains the speed-up in naming time overall and the specificity of this effect to particular objects. Furthermore, within the connectionist framework in which Hinton's model has been outlined, we could imagine that a change in the resting level of canonical object representations could result not only in generally faster response times, but also in a reduction of the orientation effect. This interactive effect could occur due to the topdown influence that canonical representations have on the activation levels of the mapping units. In this view, therefore, smaller orientation effects would be expected for objects whose canonical representation have higher resting activation levels. Unfortunately, without a more fully worked out model or a simulation of the model, such as that proposed by McClelland and Rumelhart (1981) for word recognition, it is unclear what precise predictions derive from Hinton's (1981) model. It can be suggested that, in principle, the approach proposed by Hinton (1981) is not inconsistent with the specificity of the reduction in the orientation effect.

Although the identification of patterns in novel orientations cannot be based only on orientation-invariant features, it is possible that the reduction in the effect of orientation observed across blocks of trials in this research could be due to such features. With repeated presentations, subjects could learn to associate particular patterns with distinguishing attributes of the object (e.g., a particular color), which could later reduce the impact of orientation on recognition time. Presumably subjects would learn gradually what features in fact distinguish among the patterns, which allows us to predict that the orientation effect should decrease gradually with repeated exposures of the patterns.

A final issue raised by the present results is that the magnitude of the orientation effect does not change with practice in the left/right task (Experiment 4), but it does for perceptual identification (Experiments 1-3), and that the original effect of orientation is equivalent in the two tasks (for orientations between $0^{\circ}$ and $120^{\circ}$, see Figure 6). In a template model, we can assume that left/right decisions are made at the orientation required by the response (i.e., at $0^{\circ}$ ) and thus require mental rotation. Identifying the object per se (e.g., by rotating the input to the orientation of a previously seen view of the object) would not allow the determination of the direction of pointing, and thus subjects always mentally rotate the pattern to the upright before making the left/right decision.

In the Marr and Nishihara (1978) model, it is not clear why orientation should have a linear effect on left/right decisions (or on naming times, as discussed above). One solution is that left/right decisions involve a fundamentally different problem than that posed by perceptual identification. Perceptual identification, in this model, requires making contact between a perceptual input and a stored object-centered representation. Left/right decisions, in contrast, may require an additional step in which the relationship between the object-centered frame of reference used to identify the object and a viewer-centered frame of reference must be used to make the decision. Mental rotation may be the process that relates object-centered and viewer-centered frames of reference.

In Hinton's model, we can assume that left/right decisions require that mapping units have a "handedness" (Hinton \& Parsons, 1981). Thus, some mapping units are left-handed (i.e., map objects that point left into the appropriate canonical representation) and some units are right-handed. According to Hinton and Parsons (1981), people do not know the handedness of the mapping unit assigned to a figure during the identification process. They argue that the function of mental rotation is to relate the orientation of the mapping unit with the orientation of some other frame of reference available to the subject (e.g., retinal coordinates). (This argument is similar to that proposed above in the context of the Marr and Nishihara model). If we adopt this position, however, it is not clear why initial perceptual identification and left/right decisions should be affected equally tification and left/right decisions should be affected equally by rotating the stimuli over a wide range of orientations (see Figure 6). If the orientation effect in perceptual identification is due to differences in the resting levels of mapping units tuned to different orientations, and mental rotation is required to make left/right decisions after identification has occurred, then the orientation effect on left/right decisions should be much larger than that found on identification. Given that Hinton and Parsons (1981) argue that mental rotation is a process available to subjects, a solution to the present problem is to assume that mental rotation is used for initial identifications, when subjects are not familiar with the objects. After some exposure to the objects, however, the resting activation levels for these objects may be sufficiently elevated to allow identification without mental rotation, as in the scheme originally proposed by Hinton (1981).

In conclusion, the present results suggest that mental rotation may be involved in the identification of line drawings of natural objects seen for the first time. However, processes other than mental rotation must be postulated 
to account for the nonlinear orientation effect on naming time and the reduction of the orientation effect with practice. The exact nature of these other processes remains to be elucidated.

\section{REFERENCES}

Bartram, D. J. (1974). The role of visual and semantic code in object naming. Cognitive Psychology, 6, 325-356

Corballis, M. C., \& Nagourney, B. A. (1978). Latency to categorize disoriented alphanumeric characters as letters or digits. Canadian Journal of Psychology, 32, 186-188.

Corballis, M. C., ZbrodofF, N. J., \& Roldan, C. E. (1976). What's up in mental rotation? Perception \& Psychophysics, 19, 525-530.

Corballis, M. C., Zbrodoff, N. J., Shetzer, L. I., \& Butler, P. B. $\left(19^{7} \therefore\right.$ Decisions about identity and orientation of rotated letters and digits. Memory \& Cognition, 6, 98-107.

Dearborn, G. V. N. (1899). Recognition under objective reversal. Psychological Review, 6, 395-406

Dixon, P., \& JUST, M. A. (1978). Normalization of irrelevant dimensions in stimulus comparisons. Journal of Experimental Psychology: Human Perception \& Performance, 4, 36-46.

Eley, M. G. (1982). Identifying rotated letter-like symbols. Memory \& Cognition, 10, 25-32.

FITTS, P. M., \& SEEgER, C. M. (1953). S-R compatibility: Spatial characteristics of stimulus and response codes. Journal of Experimental Psychology, 46, 199-210.

Gibson, E. (1969). Principles of perceptual learning and development. New York: Appleton-Century-Crofts.

Gibson, J. J., \& RoBinson, D. (1935). Orientation in visual perception: The recognition of familiar plane forms in differing orientations. Psychological Monographs, 46, 39-47.

Hinton, G. E. (1981). A parallel computation that assigns canonical object-based frames of reference. In Proceedings of the Seventh Intemational Joint Conference on Artificial Intelligence, Vol. 2, (pp. 683-685). Los Altos: Kaufman.

Hinton, G. E., Parsons, L. M. (1981). Frames of reference and mental imagery. In A. Baddely \& J. Long (Eds.), Attention and performance: $I X$. Hillsdale, NJ: Erlbaum.

JACOBY, L. L., \& DALLAS, M. (1981). On the relationship between autobiographical memory and perceptual learning. Journal of Experimental Psychology: General, 110, 306-340.

JoHNston, J. C. (1981). Understanding word perception: Clues from studying the word-superiority effect. In O. J. L. Tzeng \& H. Singer (Eds.), Perception of print: Reading research in experimental psychology. Hillsdale, NJ: Erlbaum.

Jolicoeur, P., GluCK, M. A., \& Kosslyn, S. M. (1984). From pictures to words: Making the connection. Cognitive Psychology, 16, 243-275.

Jolicoeur, P., \& Kosslyn, S. M. (1983). Coordinate systems in the long-term memory representation of three-dimensional shapes. $\mathrm{Cog}$ nitive Psychology, 15, 301-345.

JolicoeUR, P., \& LANDAU, M. J. (1984). Effects of orientation on the identification of simple visual patterns. Canadian Joumal of Psychology, 38, 80-93.
Kolers, P. A., \& Perkins, D. N. (1969a). Orientation of letters and errors in their recognition. Perception \& Psychophysics, 5, 265-269.

Kolers, P. A., \& Perkins, D. N. (1969b). Orientation of letters and their speed of recognition. Perception \& Psychophysics, 5, 275-280.

Koriat, A., \& Norman, J. (1984). What is rotated in mental rotation? Journal of Experimental Psychology: Learning, Memory, \& Cognition, 10, 421-434.

Koriat, A., \& Norman, J. (1985). Reading rotated words. Journal of Experimental Psychology: Human Perception \& Performance. 11, 490-508.

MARR, D. (1982). Vision. San Francisco: Freeman.

MARR, D., \& Nishihara, H. K. (1978). Representation and recognition of the spatial organization of three-dimensional shapes. Proceedings of the Royal Society of London, Series B, 200, 269-294.

MCClelland, J. L., \& RuMElHarT, D. E. (1981). An interactive activation model of context effects in letter perception: Part I. An account of basic findings. Psychological Review, 88, 375-407.

NeIsSer, U. (1967). Cognitive Psychology. New York: AppletonCentury-Crofts.

REED, S. K. (1978). Schemes and theories of pattern recognition. In E. C. Carterette \& M. P. Friedman (Eds.), Handbook of perception, Volume IX: Perceptual Processing. New York: Academic Press.

ReEd, S. K. (1982). Cognition. Belmont, CA: Wadsworth.

Rock, I. (1956). The orientation of forms on the retina and in the environment. The American Journal of Psychology, 69, 513-528.

Rock, I. (1973). Orientation and form. New York: Academic Press.

Rock, I. (1974). The perception of disoriented figures. Scientific American, 230, 78-85.

Rock, I., DiVita, J., \& Barbeito, R. (1981). The effect on form perception of change of orientation in the third dimension. Journal of Experimental Psychology: Human Perception \& Performance, 7, 719-732

RUMELHART, D. E. (1970). A multicomponent theory of the perception of briefly exposed visual displays. Journal of Mathematical Psychology, 7, 191-218.

SHWARTZ, S. P. (1981). The perception of disoriented complex objects. Unpublished manuscript, Yale University.

ShePARD, R. N., \& CoOper, L. A. (1982). Mental images and their transformations. Cambridge, MA: The MIT Press.

ShePARD, R. N., \& MetzleR, J. (1971). Mental rotation of threedimensional objects. Science, 171, 701-703.

Simion, F., Bagnara, S., Roncato, S., \& Umilta, C. (1982). Transformation processes upon the visual code. Perception \& Psychophysics, 31, 13-25

SNODGrass, J. G., \& VANDERWART, M. (1980). A standardized set of 260 pictures: Norms for name agreement, image agreement, familiarity, and visual complexity. Journal of Experimental Psychology: $\mathrm{Hu}$ man Learning \& Memory, 6, 174-215.

SPERLING, G. (1963). A model for visual memory tasks. Human Factors, $5,19-31$.

SPOEHR, K. T., \& LehMKUhle, S. W. (1982). Visual information processing. San Francisco: Freeman.

WARRINGTON, E. K., \& TAYLOR, A. M. (1973). The contribution of the right parietal lobe to object recognition. Cortex, 9, 152-164.

WhiTE, M. J. (1980). Naming and categorization of tilted alphanumeric characters do not require mental rotation. Bulletin of the Psychonomic Society, 15, 153-156.

\section{APPENDIX A}

\begin{tabular}{ll}
\hline Category & \multicolumn{1}{c}{ Objects } \\
Dog & collie, beagle, retriever, poodle, dachsund, afghan \\
Bird & canary, dove, robin, penguin, ostrich, peacock \\
Boat & speedboat, sailboat, rowboat, raft, submarine, tugboat \\
Fruits \& & \\
Vegetables & apple, pear, pumpkin, pineapple, green pepper \\
Furniture & table, chair, sofa, stove, refrigerator \\
Clothing & pants, dress, t-shirt, glove, hat \\
Car & station wagon, Jeep, Rolls Royce \\
\hline
\end{tabular}

Note-This appendix contains the names of the items used in Experiment 1 and the categories from which they were selected. 
APPENDIX B

\begin{tabular}{|c|c|c|c|c|c|c|c|}
\hline Object & Familiarity & Object & Familiarity & Object & Familiarity & Object & Familiarity \\
\hline *Alligator & 1.65 & Cup & 4.40 & $*$ Iron & 3.65 & *Squirrel & 3.35 \\
\hline Basket & 2.18 & *Deer & 2.22 & *Kangaroo & 1.92 & Stove & 4.65 \\
\hline *Bear & 1.98 & $*$ Dog & 4.60 & $*$ Kettle & 3.80 & Suitcase & 3.65 \\
\hline Bed & 4.72 & *Donkey & 1.88 & Lamp & 4.20 & *Swan & 1.97 \\
\hline Bell & 2.20 & Dresser & 4.52 & *Leopard & 1.92 & Sweater & 4.48 \\
\hline Bottle & 3.72 & Eagle & 2.42 & $*$ Lion & 2.00 & Table & 4.35 \\
\hline Bowl & 4.18 & *Elephant & 2.35 & *Ostrich & 1.52 & Television & 4.82 \\
\hline Bread & 4.40 & *Foot & 4.78 & Owl & 2.22 & Telephone & 4.80 \\
\hline *Bus & 4.50 & $*$ Fox & 1.95 & Pants & 4.55 & *Tiger & 2.10 \\
\hline Cake & 4.02 & $*$ Frog & 2.48 & *Penguin & 1.70 & Toaster & 4.08 \\
\hline *Cannon & 1.52 & Garbage can & 4.08 & $*$ Raccoon & 2.20 & *Train & 4.15 \\
\hline *Car & 4.70 & *Giraffe & 1.80 & Record player & 4.40 & Tree & 4.68 \\
\hline *Cat & 4.22 & Glass & 4.78 & $*$ Rhinoceros & 1.52 & *Truck & 4.02 \\
\hline Chair & 4.58 & *Goat & 2.05 & *Rooster & 2.22 & *Turtle & 2.40 \\
\hline *Chicken & 2.42 & *Gorilla & 2.05 & *Sheep & 1.85 & Well & 1.45 \\
\hline Clock & 4.38 & Grasshopper & 2.42 & Shirt & 4.56 & Windmill & 1.80 \\
\hline Couch & 4.40 & Harp & 1.88 & *Skunk & 2.30 & Wineglass & 4.02 \\
\hline Crown & 1.52 & House & 4.38 & Spider & 2.28 & *Zebra & 1.60 \\
\hline
\end{tabular}

Note-This appendix lists the names of the items used in Experiments 2 to 4 and their mean familiarity rating (on a 5-point scale with I indicating very unfamiliar; see Snodgrass \& Vanderwart, 1980). *Item used in Experiment 4.

(Manuscript received April 23, 1984;

revision accepted for publication March 25, 1985.) 